Classification

Physics Abstracts

$74.10-74.70-05.90$

\title{
Superconducting diamagnetism near the percolation threshold
}

\author{
R. Rammal (*), T. C. Lubensky $\left({ }^{* *}\right)$ and G. Toulouse \\ Laboratoire de Physique de l'ENS, 24, rue Lhomond, 75231 Paris Cedex 05, France
}

(Reçu le 23 août 1982, accepté le $1^{\mathrm{er}}$ décembre 1982)

\begin{abstract}
Résumé. - On présente des lois d'échelle pour la susceptibilité diamagnétique $\bar{\chi}$, et pour le champ critique supérieur- $H_{\mathrm{c} 2}$, de mélanges aléatoires de composants supraconducteurs et isolants. En dimension deux, l'exposant critique qui apparaît dans cette théorie est relié à d'autres exposants de percolation. Les prédictions qui en résultent pour $H_{\mathrm{c} 2}$ sont confrontées à des données expérimentales sur des films InGe.
\end{abstract}

Abstract. - A scaling theory for the diamagnetic susceptibility, $\bar{\chi}$, and the upper critical field, $H_{\mathrm{c} 2}$, of random mixtures of superconducting and insulating elements is presented. In two dimensions, the new critical exponent in this theory is related to other percolation exponents. The resulting predictions for $H_{\mathrm{c} 2}$ are compared with experiments on InGe films.

Consider a random superconductor-insulator mixture. The diamagnetic properties of such a mixture and their variation across the percolation threshold (for the superconducting component) raise an interesting problem.

Far on the metallic side [1] $\left(p \gg p_{\mathrm{c}}\right)$, the zero field diamagnetic susceptibility has a jump at the superconducting transition temperature $T_{0}$. (At this stage, and later, we ignore any fluctuation effects : the superconducting transition is treated within mean-field theory, the emphasis being on the geometrical effects associated with disorder). On the insulating side ( $\left.p<p_{\mathrm{c}}\right)$, the susceptibility increases linearly with temperature below $T_{0}$ (Fig. 1). One question is : how does the system evolve from one behaviour to the other ? Otherwise stated, is the infinite percolation cluster at threshold $\left(p=p_{c}\right)$ dense enough to give rise to a Meissner effect ? Questions of this kind were raised by de Gennes [2,3] in connection with experiments done in Tel-Aviv [4], measuring the behaviour of the critical field, $H_{\mathrm{c} 2}$, of InGe films in the vicinity of $T_{0}$. As is the case for the susceptibility, there is a problem of crossover for the critical field, which is expected to rise as a function of temperature, $T$, with finite slope on the metallic side and with infinite slope on the insulating side (Fig. 2). The slope, $\mathrm{d}_{\mathrm{c} 2} / \mathrm{d} T$, measured on the metallic side, was found to diverge, for $p \rightarrow p_{c}$, as :

$$
\frac{\mathrm{d} H_{\mathrm{c} 2}}{\mathrm{~d} T} \sim \frac{1}{\left(p-p_{\mathrm{c}}\right)^{k}}
$$

with $k=0.6 \pm 0.05$. Thus the question of the theoretical origin of this exponent arises.

(*) Permanent address : C.R.T.B.T., B.P. 166, 38042 Grenoble, France.

$\left({ }^{* *}\right)$ Permanent address : Department of Physics, University of Pennsylvania, Philadelphia, PA 19104, U.S.A. 


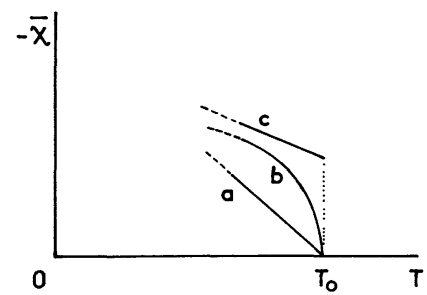

Fig. 1. - Diamagnetic susceptibility $\bar{\chi}$ as a function of temperature $T$, for a very large sample : a) $p<p_{\mathrm{c}}$ (finite clusters); b) $p=p_{\mathrm{c}}$; c) $p>p_{\mathrm{c}}$ (bulk behaviour).

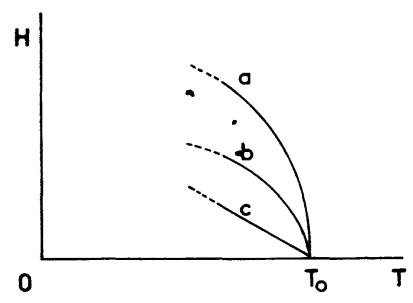

Fig. 2. - Upper critical field $H_{\mathrm{c} 2}$, in the same three regimes as in figure 1.

This paper proposes a theoretical description which attempts to answer these various questions in a simple unifying way. We present predictions for the two-dimensional problem which differ rather sharply from those made by previous workers $[4,5]$. This is a rather nascent field which, it is fair to say, calls for further experimental and theoretical study (in particular, concerning the three-dimensional case).

1. The model. - We consider a two-dimensional mixture of superconducting and insulating grains in a perpendicular magnetic field.

Following de Gennes [2], we describe the superconducting clusters as ramified networks of wires with elementary grain sizes (which determine the transverse width of the wires) that are smaller than the London penetration length $\lambda$. The diamagnetic susceptibility per unit volume of superconducting matter, $\bar{\chi}$, of such a cluster can then be expressed $[2,5]$ as :

$$
\bar{\chi}=-\frac{S^{\text {eff }}}{16 \pi^{2} \lambda^{2}},
$$

where $S^{\text {eff }}$, the effective area, is a geometrical quantity which contains information about the topology of the cluster. Since $\lambda$, in the vicinity of the transition temperature $T_{0}$, varies as :

$$
\lambda \sim(\Delta T)^{-1 / 2}, \quad \Delta T=T_{0}-T,
$$

the diamagnetic susceptibility of a finite cluster is predicted to increase linearly with $T$ below $T_{0}$. If one considers now a collection of such finite clusters, as exists on the insulating side of a percolation threshold, $p<p_{c}$, the total diamagnetic susceptibility may be taken as the sum of the contributions of each cluster, provided one restricts one's attention to temperatures close enough to $T_{0}$ that the effects of dipolar interactions between clusters remain neglibible. Thus, on the insulating side of the percolation threshold, the diamagnetic susceptibility is expected to 
vary linearly in $\Delta T$. The question is whether its slope, which is a function of $\left(p_{c}-p\right)$, diverges when $p \rightarrow p_{\mathrm{c}}$.

On the metallic side $\left(p>p_{c}\right)$ in finite samples (linear length $\left.L\right)$, the same argument indicates that :

$$
\bar{\chi} \sim-(\Delta T) L^{2}
$$

the coefficient being a function of $p$.

2. Scaling laws. - We can assemble all available information about this problem by writing a scaling expression for $\bar{\chi}$. For this purpose, we first recognize that there are at least three lengths of importance in this problem :

i) the length of the sample, $L$,

ii) the superconducting coherence and penetration lengths, $\xi_{\mathrm{s}}$ and $\lambda$, which both diverge in the same manner (3), as the superconducting transition temperature is approached,

iii) the percolation correlation length, $\xi_{\mathrm{p}}$, which diverges as the percolation threshold is approached :

$$
\xi_{\mathrm{p}} \sim|\Delta p|^{-v}, \quad \Delta p=p-p_{\mathrm{c}} .
$$

There is another (microscopic) length, namely the grain size $a$; but in the region of the phase diagram which is of interest here (vicinity of the transition temperature around percolation threshold), $a$ is much smaller than the three previously quoted lengths and may be ignored.

With this in mind, we hypothesize that the diamagnetic susceptibility, per unit volume of superconducting matter, $\bar{\chi}$, obeys a scaling law of the form :

$$
\bar{\chi} \sim-|\Delta p|^{\phi} f\left(\frac{L}{\xi_{\mathrm{s}}}, \frac{L}{\xi_{\mathrm{p}}}\right),
$$

where $\phi$ is some exponent. Formula (6) can equivalently be written as :

$$
\bar{\chi} \sim-\xi_{\mathrm{p}}^{-\bar{\delta}} f\left(\frac{L}{\xi_{\mathrm{s}}}, \frac{L}{\xi_{\mathrm{p}}}\right)
$$

where $\phi=v \bar{\delta}$. This scaling law assumes simple forms in the five regions of the $p-T$ phase diagram, defined on figure 3 , which are separated by crossover boundaries, corresponding to equalities of two out of the three basic lengths. Boundary 1 corresponds to $\xi_{\mathrm{s}} \sim \xi_{\mathrm{p}}$, boundary 2 to $L \sim \xi_{\mathrm{s}}$, boundary 3 to $L \sim \xi_{\mathrm{p}}$.

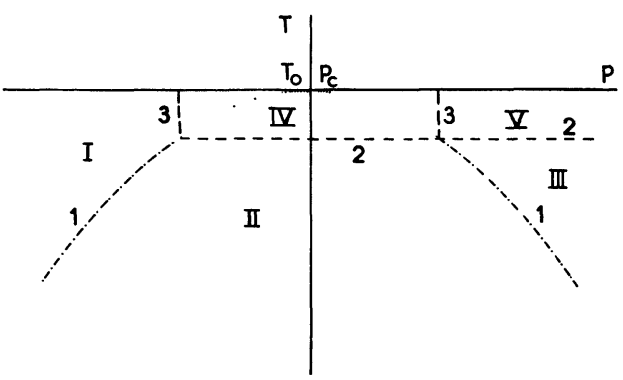

Fig. 3. $-p-T$ phase diagram around the transition temperature $\left(T=T_{0}\right)$ and the percolation threshold $\left(p=p_{\mathrm{c}}\right.$ ) for a random superconductor-insulator mixture of finite size $L$. Definitions for the regions I to $\mathrm{V}$ and for the boundaries 1 to 3 are given in the text. 
In region $\mathrm{I}, \xi_{\mathrm{p}} \ll\left(\xi_{\mathrm{s}}, L\right)$ and $\bar{\chi}$ should be independent of $L$ and linear in $\Delta T$ because the system is an assembly of small clusters :

$$
-\bar{\chi} \sim \xi_{\mathrm{s}}^{-2} \xi_{\mathrm{p}}^{2-\bar{\delta}} \sim(\Delta T)(\Delta p)^{-v(2-\bar{\delta})} .
$$

In region $\mathrm{IV}, L \ll\left(\xi_{\mathrm{s}}, \xi_{\mathrm{p}}\right)$ and $\bar{\chi}$ should be independent of $\xi_{\mathrm{p}}$ and linear in $\Delta T$ :

$$
-\bar{\chi} \sim \xi_{\mathrm{s}}^{-2} L^{2-\bar{\delta}} \sim(\Delta T) L^{2-\bar{\delta}} .
$$

In region $\mathrm{V}, \xi_{\mathrm{s}} \gg L \gg \xi_{\mathrm{p}}$ and the behaviour corresponding to equation (4) holds :

$$
-\bar{\chi} \sim \xi_{\mathrm{p}}^{-\bar{\delta}} L^{2} \xi_{\mathrm{s}}^{-2} \sim(\Delta T) L^{2}\left(p-p_{\mathrm{c}}\right)^{v \bar{\delta}} .
$$

In regions II and III, a bulk regime is reached and the $L$ dependence disappears. Region II corresponds to $\xi_{\mathrm{s}} \ll\left(L, \xi_{\mathrm{p}}\right)$, and $\bar{\chi}$ is a function of $\xi_{\mathrm{s}}($ or $\Delta T)$ only :

$$
-\bar{\chi} \sim \xi_{\mathrm{s}}^{-\bar{\delta}} \sim \cdot(\Delta T)^{\bar{\delta} / 2}
$$

Finally, region III corresponds to $\xi_{\mathrm{p}} \ll \xi_{\mathrm{s}} \ll L$ and $\bar{\chi}$ is a function of $\xi_{\mathrm{p}}$ (or $\Delta p$ ) only :

$$
-\bar{\chi} \sim \xi_{\mathrm{p}}^{\bar{\delta}} \sim(\Delta p)^{\nu \bar{\delta}} \text {. }
$$

Our scaling hypothesis for $\bar{\chi}$, equation (7), plus previously given (section 1 ) information for the susceptibility of finite clusters, has thus allowed us to reach a rather detailed set of predictions for the behaviour of $\bar{\chi}$. All exponents are expressed in terms of one unknown $\bar{\delta}$. The exponent $v$ is the two-dimensional correlation length exponent, which is now widely believed to be exactly $4 / 3$ [10].

The question raised by de Gennes [2] concerning the divergence of the slope of $\bar{\chi}$ on the insulating side (8) amounts to asking whether $\bar{\delta}$ is smaller or larger than 2 . In order to determine the value of $\delta$, we shall recall some recent results [5-7] bearing on the size dependences of the diamagnetic susceptibility, $\bar{\chi}$, and of the resistance, $R$, for a self-similar structure called the Sierpinski gasket. It has been found that :

$$
-\bar{\chi} \sim(\Delta T) L^{2-\bar{\delta}}
$$

and

$$
R \sim L^{2+\bar{\delta}-\bar{d}},
$$

where $\bar{d}$ is the fractal dimensionality of the gasket and $\bar{\delta}$ represents the same number in (13) and (14).

We now assume that relations (13) and (14), rigorously proven only for a special family of fractal objects, have a more general validity and still hold, in particular, for the percolation cluster at threshold which is also a fractal object. This generalization, which is not unreasonable, and which has already been assumed by S. Alexander and R. Orbach [8] in a different context, leads to predictions which have the merit of being testable experimentally and numerically, as discussed later. In the context of percolation,

$$
\bar{d}=d-\frac{\beta}{v}
$$

where, here, $d=2$ and $\beta$ is the percolating cluster density exponent. Equation (14) leads then to the relation :

$$
v \bar{\delta}=t-\beta
$$

where $t$ is the percolation conductivity exponent. 
Since $\beta$ and $t$ are presently determined with rather high accuracy $[7,9,10]$ :

$$
\beta \simeq 0.14, \quad t \simeq 1.28,
$$

this leads to the prediction (see also Ref. [8]) :

$$
\bar{\delta} \simeq 0.85 \text {. }
$$

Consequently, the slope of $\bar{\chi}$ on the insulating side, equation (8), is predicted to diverge at the percolation threshold as

$$
-\frac{\bar{\chi}}{\Delta T} \sim(\Delta p)^{-(2 v-t+\beta)}
$$

with $2 v-t+\beta \simeq 1.53$. This is in sharp contrast with the analysis of reference [5], concluding that this slope would not diverge.

3. Comparison with numerical or experimental results. - As formulated above, the scaling law for $\bar{\chi}$ as a function of $\xi_{\mathrm{p}}, \xi_{\mathrm{s}}$ and $L$ can be tested numerically. A quantity which is directly computable is the effective area, $S$ eff , in situations with finite size clusters or sample. This corresponds to regions $\mathrm{I}, \mathrm{IV}, \mathrm{V}$ for which our predictions can be resummarized as follows :

i) at $p=p_{\mathrm{c}}, S^{\text {eff }}\left(p_{\mathrm{c}}\right)$ reduces to a power law function of the sample length $L$ :

$$
S^{\text {eff }}\left(p_{\mathrm{c}}\right) \sim L^{2-\bar{\delta}}
$$

ii) in the region $p>p_{\mathrm{c}}$, and $L \gg \xi_{\mathrm{p}}$, one has

$$
\frac{S^{\mathrm{eff}}(p)}{S^{\mathrm{eff}}(p=1)} \sim\left(p-p_{\mathrm{c}}\right)^{v \bar{\delta}}
$$

iii) below $p_{c}, S^{\text {eff }}(p)$ is predicted to show the following behaviour :

$$
S^{\text {eff }}(p) \sim\left(p_{\mathrm{c}}-p\right)^{-v(2-\bar{\delta})} .
$$

Recent numerical experiments [11], using de Gennes [2] formula for $S^{\text {eff }}$, lend strong support to the above predicted scaling laws. Monte Carlo calculations, on finite square lattice samples, also permit the value of $\bar{\delta}$ to be calculated. The numerical value so obtained, $\bar{\delta}=0.79 \pm 0.02$, is in satisfactory agreement with the predicted value equation (18). In addition, one observes that the finite size effects close to $p_{c}$ are in good accord with the scaling laws.

From the behaviour of the diamagnetic susceptibility, described in section 2, the behaviour of $H_{\mathrm{c} 2}$ can be derived. This leads to a second series of predictions which can be compared with experimental data, some of which are already available [4].

The correspondence between $\bar{\chi}$ and $H_{\mathrm{c} 2}$ is based on the relation :

$$
-\bar{\chi} H_{\mathrm{c} 2}^{2} \sim(\Delta T)^{2}
$$

which is a direct consequence of a mean-field treatment of the superconducting transition [1]. Such a relation is clearly satisfied on both sides of the percolation threshold (curves a and $\mathrm{c}$ of Figs. 1 and 2). It allows us to derive expressions for $H_{\mathrm{c} 2}$ in regions I, II, III. We shall leave aside finite size effects since we want to make a comparison with experiments, which are performed on large size two-dimensional films. 
In region $\mathrm{I}$, one finds :

$$
H_{\mathrm{c} 2}^{2} \sim(\Delta T)(\Delta p)^{v(2-\bar{\delta})},
$$

with an $H_{\mathrm{c} 2}$ increasing with vertical slope, for $p<p_{\mathrm{c}}$.

In region II, $H_{\mathrm{c} 2}$ is independent of $\Delta p$ :

$$
H_{\mathrm{c} 2}^{2} \sim(\Delta T)^{2-\bar{\delta} / 2} .
$$

In region III, $H_{\mathrm{c} 2}$ has a bulk behaviour with a linear variation in $\Delta T$ :

$$
H_{\mathrm{c} 2}^{2} \sim(\Delta T)^{2}(\Delta p)^{-v \bar{\delta}} .
$$

The latter law provides a prediction for the exponent $k$, defined in the introduction :

$$
k=\frac{v \bar{\delta}}{2}=\frac{t-\beta}{2} \text {. }
$$

Using the values given in section 2 for $\bar{\delta}$ and $v$, this yields a prediction :

$$
k \simeq 0.57
$$

in satisfactory agreement with the experimental data of the Tel-Aviv group [4].

In reference [4], an estimate of $k$ was given whereby :

$$
k=t+\beta-\beta^{\prime}
$$

where $\beta^{\prime}$ is the backbone density exponent [12]. Using the presently accepted values for $t, \beta$ and $\beta^{\prime}\left(\beta^{\prime} \simeq 0.55\right)$, this yields $k \simeq 0.87$, which is in less good accord with the existing data [13]. It is worth noting that our theory does not make explicit use of the notion of the backbone of the infinite cluster, in contrast with some of the other theories $[4,5]$ of magnetic superconducting properties. Of course, it may be that there exists a relation between exponent $\beta^{\prime}$ and exponent $t$, which would make the various theories more directly comparable.

4. Conclusion. - We have presented a scaling theory for the magnetic properties of random mixtures of superconducting and insulating elements. This theory involves the three lengths $\xi_{\mathrm{s}}$, $\xi_{\mathrm{p}}$ and $L$, and one new exponent $\bar{\delta}$. In two dimensions, we argue that $\bar{\delta}$ can be expressed in terms of the conductivity and percolating cluster exponents, $t$ and $\beta$, via equation (16). The resulting prediction for the behaviour of $\mathrm{dH}_{\mathrm{c} 2} / \mathrm{d} T$ on the superconducting side is in good agreement with experiments on InGe films. Our scaling hypothesis leads to other predictions which varrant experimental investigation. In particular, it would be desirable to have any data in three dimensions. Furthermore, investigations of the anomalous temperature dependence of $H_{\mathrm{c} 2}$ near the percolation threshold in both two and three dimensions would be of interest. On the theoretical side, though the existence of a scaling law in three dimensions appears plausible, it is not clear at all whether the exponent $\bar{\delta}$ in this case is simply related to other standard percolation exponents.

Our analysis, despite its good agreement with the existing data, is perhaps oversimplified. It may well be that further experimental and numerical activity will require a theory of a more refined level than appears useful today.

Acknowledgments. - We thank R. Orbach for numerous stimulating discussions and for making available reference [8] prior to publication. We are also grateful to J. C. Angles d'Auriac, S. Alexander and J. Vannimenus for helpful comments. One of us (T.C.L.) acknowledges financial support from the John Simon Guggenheim Foundation and from O.N.R. under contract N000140106 and N.S.F. under contract DMR 79-1013. 


\section{References}

[1] Tinkham, M., Introduction to Superconductivity (McGraw-Hill) 1975.

[2] De Gennes, P. G., C. R. Hebd. Séan. Acad. Sci., Série II 292 (1981) 9.

[3] De Gennes, P. G., C. R. Hebd. Séan. Acad. Sci., Série II 292 (1981) 279.

[4] Deutscher, G., Grave, I. and Alexander, S., Phys. Rev. Lett. 48 (1982) 1497 ; Deutscher, G., Lecture Notes in Physics vol. 149 (Springer) 1981, p. 26.

[5] Stephen, M. J., Phys. Lett. 87A (1981) 67.

[6] Alexander, S., Jerusalem preprint (1981), submitted to J. Low Temp. Phys.

[7] Gefen, Y., Aharony, A., Mandelbrot, B. B. and Kirkpatrick, S., Phys. Rev. Lett. 47 (1981) 1771.

[8] Alexander, S. and Orbach, R., J. Physique-Lett. 43 (1982) L-625.

[9] Stauffer, D., Phys. Rep. 54 (1979) 1.

[10] Derrida, B. and Vannimenus, J., J. Phys. $A 15$ (1982) L-557.

[11] Rammal, R. and Angles D'Auriac, J. C., C.R.T.B.T. preprint (1982).

[12] KirkPATRICK, S., in Ill-condensed Matter, Eds. R. Balian, R. Maynard, G. Toulouse (North-Holland) 1979.

[13] In reference [4], a crossover in the behaviour of the critical field has been observed far from the percolation threshold, on the metallic side. This crossover lies clearly outside the range of validity of our theory which, in contrast with that of reference [4], aims only at describing the vicinity of the percolation threshold. 